\title{
Comparative Study of the Effect of Solvents on the Efficacy of Neonicotinoid Insecticides Against Malaria Vector Populations Across Africa
}

Magellan Tchouakui ( $\square$ magellan.tchouakui@crid-cam.net )

Centre for Research in Infectious Diseases https://orcid.org/0000-0002-1683-8057

\section{Tatiane Assatse}

CRID

Leon M. J. Mugenzi

CRID

Benjamin D. Menze

CRID

Daniel Nguiffo-Nguete

CRID

Williams Tchapga

CRID

Jonathan Kayondo

Uganda Virus Research Institute

Francis Watsenga

Institut National de Recherche Biomedicale

Emile Zola Manzambi

Institut National de Recherche Biomedicale

Michael Osae

Radiation entomology and pest management

Charles S. Wondji

Liverpool School of Tropical Medicine

\section{Research Article}

Keywords: malaria vectors, neonicotinoids, overestimating resistance, survive clothianidin, public health

Posted Date: January 6th, 2022

DOI: https://doi.org/10.21203/rs.3.rs-1197098/v1 
License: (c) (i) This work is licensed under a Creative Commons Attribution 4.0 International License. Read Full License

Version of Record: A version of this preprint was published at Infectious Diseases of Poverty on April 25th, 2022. See the published version at https://doi.org/10.1186/s40249-022-00962-4. 


\section{Abstract}

\section{Background}

New insecticides with a novel mode of action such as neonicotinoids have recently been recommended

for public health by WHO. Resistance monitoring of such novel insecticides requires a robust protocol to monitor the development of resistance in natural populations. In this study, we comparatively used three different solvents to assess the susceptibility of malaria vectors to neonicotinoids across Africa.

\section{Methods}

Mosquitoes were collected from May to July 2021 from three agricultural settings in Cameroon (NjombePenja, Nkolondom, and Mangoum), the Democratic Republic of Congo (Ndjili-Brasserie), Ghana (Obuasi), and Uganda (Mayuge). Using the CDC bottle test, we compared the effect of three different solvents (ethanol, acetone, MERO) on the efficacy of neonicotinoids against Anopheles gambiae s.l. In addition, TaqMan assays were used to genotype key pyrethroid-resistant markers in An. gambiae and to evaluate potential cross-resistance between pyrethroids and clothianidin.

\section{Results}

Lower mortality were observed when using absolute ethanol or acetone alone as solvent (11.4- 51.9\% mortality in Nkolondom, 31.7- 48.2\% in Mangoum, 34.6- 56.1\% in Maypge, 39.4- 45.6\% in Obuasi, 83.7$89.3 \%$ in Congo and $71.05-95.9 \%$ in Njombe pendja) compared to acetone + MERO for which $100 \%$ mortality were observed for all the populations. Synergist assays (PBO, DEM and DEF) revealed a significant increase of mortality suggesting that metabolic resistance mechanisms are contributing to the reduced susceptibility. A negative association was observed between the L1014F-kdr mutation and clothianidin resistance with a greater frequency of homozygote resistant mosquitoes among the dead than among survivors $(\mathrm{OR}=0.5 ; \mathrm{P}=0.02)$. However, the I114T-GSTe2 was in contrast significantly associated with a greater ability to survive clothianidin with a higher frequency of homozygote resistant among survivors than other genotypes $(\mathrm{OR}=2.10 ; \mathrm{P}=0.013)$.

\section{Conclusions}

This study revealed a contrasted susceptibility pattern depending on the solvents with ethanol/acetone resulting to lower mortality, thus possibly overestimating resistance, whereas the MERO consistently showed a greater efficacy of neonicotinoids but it could prevent to detect early resistance development. Therefore, we recommend monitoring the susceptibility using both acetone alone and acetone+MERO (8$10 \mu \mathrm{g} / \mathrm{ml}$ for clothianidin) to capture the accurate resistance profile of the mosquito populations.

\section{Background}

Malaria prevention relies extensively on mosquito control using pyrethroid-based interventions including Indoor Residual Spraying (IRS) and Long Lasting Insecticidal Nets [1, 2]. The scale-up of these tools has 
significantly contributed to the important reduction of malaria burden in the past decade [1,3]. However, increasing resistance to pyrethroid in malaria vector species is a serious challenge to these vector control interventions given the heavy reliance on pyrethroid only [4]. Most African An. gambiae and An. funestus populations are resistant to pyrethroid insecticides and also show varying levels of resistance to other insecticides used for vector control (carbamates, organophosphates and organochlorines). Due to the threat posed by insecticide resistance, there has been an urgent call for alternative insecticides to supplement malaria vector control [5]. Novel insecticides are gradually being introduced by manufacturers and recommended by WHO for vector control [6]. Among these, the neonicotinoids (e.g clothianidin) and Pyrole (e.g Chlorfenapyr) are the new mode of action insecticide classes for public health, recently recommended by WHO for LLINs and IRS [7].

Clothianidin is a neonicotinoid insecticide that is chemically similar to nicotine. It acts on the central nervous system of insects as an agonist of acetylcholine and stimulates nicotine acetylcholine receptors (nAChR) [8] activating post-synaptic acetylcholine receptors but does not inhibit acetyl cholinesterase (ACh). High levels overstimulate and block the receptors, [9] causing paralysis and death [8]. Clothianidin is the active ingredient in SumiShield (developed by Sumitomo Chemical Company, Japan) and Fludora ${ }^{\circledR}$ Fusion (Bayer CropScience, Monheim, Germany) along with deltamethrin, an IRS formulation which was recently added to the WHO prequalification list of recommended insecticides (https://www.who.int/pqvector-control/ prequalifed-lists/en/).

As new insecticides are developed, it is essential to monitor the development of resistance to prolong their efficacy as much as possible and avoid the level of widespread resistance now seen with pyrethroids [3]. Such resistance monitoring requires establishing diagnostic concentrations to determine baseline susceptibility of malaria vectors and to enable surveillance of insecticide resistance once the insecticides are in use.

Nowadays, WHO protocol to test the susceptibility to neonicotinoids in mosquitoes is not well established thus, making resistance monitoring to this insecticide class very challenging. Clothianidin (Neonicotinoid; IRAC MoA class 4A) for example, tends to crystallize if used in straight acetone/ ethanol (solvents commonly used in bioassays) and the uptake of active ingredient between the insect's body and the crystal is very low [10-12]. This has made the design of standard protocols more arduous compared to other insecticide classes. In addition, because this active ingredient acts slowly, resistance profiles can be detected after bioassays only if exposed populations are rigorously monitored during a long holding period, which can last seven days or more. Despite these difficulties, the susceptibility of wild Anopheles populations from several African countries has been evaluated using $150 \mu \mathrm{g} / \mathrm{ml}$ of clothianidin dissolved in either absolute ethanol or acetone [10-13]. Recently, Bayer CropScience Ltd introduced an 81\% Rapeseed oil methyl ester (1000ppm MERO ${ }^{3}$ ) which, added to acetone, prevents the crystallization then keeps the clothianidin for a longer period in a solved state and allows therefore the feasibility of the bottle assay. A study conducted in ivory coast, established $50 \mu \mathrm{g} / \mathrm{ml}$ as diagnostic dose of clothianidin [14]. However, this study did not evaluated the lower doses to see if $50 \mu \mathrm{g} / \mathrm{ml}$ cannot mask the detection of resistance highlighting the need of stabilising the protocol for testing of neonicotinoids. In this study, we 
used three different solvents to comparatively evaluate the efficacy of neonicotinoids on malaria vectors from many African countries and established the diagnostic dose of clothiandin using acetone and MERO. Furthermore, we evaluated a potential cross-resistance between pyrethroids and clothianidin.

\section{Methods}

\section{Study sites}

Mosquitoes were collected from 4 regions across the continent. Mosquitoes were collected in three agricultural settings in Cameroon (Mangoum, Nkolondom, and Njombe-Penja) from May to July 2021. The climate is made up of two wet and two dry seasons typical of tropical climate around the equator. In the Democratic Republic of Congo (DRC) mosquitoes were collected at Ndjili Brasserie, a suburb of Kinshasa ( $\left.4^{\circ} 19^{\prime} 39^{\prime \prime} \mathrm{S}, 15^{\circ} 18^{\prime} 48^{\prime \prime} \mathrm{E}\right)$, in June 2021. In Uganda, mosquitoes were collected in the Eastern region (May 2021), May $\mu$ ge $\left(0^{\circ} 23^{\prime} 10.8^{\prime \prime} \mathrm{N}, 33^{\circ} 37^{\prime} 16.5^{\prime \prime} \mathrm{E}\right)$, and in Ghana collections were done in Obuasi $\left(5^{\circ} 56^{\prime} \mathrm{N}, 1^{\circ} 37^{\prime} \mathrm{W}\right)$ in July 2021 . In Cameroon, immature stages were collected from the breeding site using the dipping method whereas in other countries indoor resting blood fed females were collected using electric aspirators. Emerging adults (2-5days old) from collected larvae or $F_{1}$ progeny (2-5days old) from indoor collected females were used for the bioassays.

\section{Molecular Identification}

The genomic DNA was extracted from a subset of mosquitoes from each of the collection sites using the Livak method [25], then the members of the An. gambiae complex was identified by the PCR [26, 27].

\section{Determination of susceptibility to neonicotinoids and establishment of the diagnostic dose of clothianidin using acetone + MERO as a solvent}

Clothianidin, imidacloprid and acetamiprid used were technical materials from Sigma (PESTANAL ${ }^{\circledR}$, analytical standard, Sigma-Aldrich, Dorset, United Kingdom). These chemicals were mixed in three different solvents (acetone, absolute ethanol, or acetone with MERO). For acetone and ethanol alone, the dose of $150 \mu \mathrm{g} / \mathrm{ml}$ for clothianidin, $200 \mu \mathrm{g} / \mathrm{ml}$ for imidacloprid and $75 \mu \mathrm{g} / \mathrm{ml}$ for acetamiprid were used as previously described [11].

For acetone dissolved in MERO, a stock solution of an acetone/MERO® mixture was made by pipetting $0.11 \mathrm{ml}(110 \mu \mathrm{l}) \mathrm{MERO} \circledast$ to $100 \mathrm{ml}$ of acetone $(\sim 1000 \mathrm{ppm}) .900 \mu \mathrm{g}$ straight clothianidin was weighed and mixed with $10 \mathrm{ml}$ of this stock solution of MERO plus acetone. After complete dissolution of the active ingredient, $10 \mathrm{ml}$ acetone/MERO® solution containing $900 \mu \mathrm{g}$ clothianidin was prepared. One (1) $\mathrm{ml}$ of this solution was applied to each test bottle to achieve a concentration of $90 \mu \mathrm{g}$ clothianidin $/ 250 \mathrm{ml}$ bottle. This dose of insecticide was used to characterise the susceptibility of different mosquito populations. In addition, ranges of insecticide concentrations were tested $(0.25,0.50,1,2,4,40$ and 90 $\mu \mathrm{g} / \mathrm{ml}$ ) using the susceptible lab strain Kisumu to evaluate the diagnostic dose of clothianidin when diluted in acetone and MERO. Approximately $24 \mathrm{~h}$ after coating bottles with insecticide, 25 female Kisumu 
(3-5 days old) were exposed to the insecticides for $1 \mathrm{~h}$ and the knocked down mosquitoes were recorded at the end of the $60 \mathrm{~min}(\mathrm{Kd}-60)$ exposure period. After recording the $\mathrm{Kd}-60$ mosquitoes were gently aspirated from the bottle into clean paper cups and provided with $10 \%$ sugar solution soaked in cotton wool during the recovery period and the final mortality was recorded $24 \mathrm{~h}$ post-exposure.

\section{Synergist assay with piperonyl butoxide (PBO), di-ethyl Maleate (DEM) and s,s,s-tri- butylphosphorotrithioate (DEF)}

To identify the possible enzyme systems involved in reduced susceptibility to neonicotinoids, synergist bioassays were conducted for clothianidin in Nkolondom using the emerging adult from larval collection. Two- to four-day-old $F_{0} / F_{1}$ females were first exposed to the synergist ( $4 \% \mathrm{PBO}, 8 \% \mathrm{DEM}$ or $0.25 \% \mathrm{DEF}$ ) for $1 \mathrm{~h}$, followed by exposure to $150 \mu \mathrm{g} / \mathrm{ml}$ clothianidin (dissolved in acetone) for $1 \mathrm{~h}$. Mortality was recorded $24 \mathrm{~h}$ after exposure and the differences in mortalities between synergized and non-synergized experiments were compared using a Chi-square test.

\section{Potential cross-resistance between neonicotinoids and pyrethroids}

To assess the potential cross-resistance between new neonicotinoids and pyrethroids, we crossed the pyrethroid highly resistant field strain from Nkolondom (where the 1014F-Kdr is fixed) with the fully susceptible laboratory strain Kisumu (with no 1014F-Kdr). This hybrid strain was exposed to sub-lethal doses of clothianidin (dissolved in acetone only) to select the dead and alive mosquitoes. These mosquitoes were genotyped for the L1014F target-site knockdown resistance (Kdr_w) and the 114TGSTe2 metabolic resistance marker (all associated with DDT/pyrethroid resistance in An. gambiae) using Taqman methods as previously described $[15,16]$. PCR reactions $(10 \mu \mathrm{l})$ contained $1 \mu \mathrm{l}$ of genomic DNA, $5 \mu$ l of SensiMix DNA kit (catalog: SM2-717104), $0.125 \mu$ l of each probe and $3.875 \mu$ l of sigma water. Samples were run on a Mx3000 $\mathrm{P}^{\mathrm{TM}}$ Multiplex quantitative PCR system with the temperature cycling conditions of: 10 minutes at $95^{\circ} \mathrm{C}$ followed by 40 cycles of $95^{\circ} \mathrm{C}$ for 10 seconds and $60^{\circ} \mathrm{C}$ for 45 seconds.

Odds ratio and Fisher exact test were used to establish the statistical significance of any association between this DDT/pyrethroid resistance marker and the ability to survive clothianidin exposure.

\section{Results}

\section{Molecular identification of mosquitoes tested}

PCR assays revealed that all the mosquitoes tested from Mangoum (44/44), Nkolondom (50/50), and Congo (60/60) were An. gambiae. Those collected in Njombe (Cameroon) were mainly An coluzzii (58/60). The An. gambiae sl population from Uganda were mix of $82 \%$ An. gambiae and $17 \% A n$. arabiensis whereas those from Ghana were 60\% (39/65) An. gambiae and 40\% (39/65) An. coluzzii.

\section{Susceptibility profile to clothianidin}


The Kisumu lab strain was susceptible to clothianidin whatever the solvent used (Figure 1A \& S1). However, the susceptibility to this insecticide varied significantly in An. gambiae field populations depending on the solvent used. The use of acetone combined with 1000ppm MERO® (81\% Rapeseed oil methyl ester) as solvent, induced significant higher mortality compared to acetone or ethanol alone

(Figure 1A). A full susceptibility was observed for all the An. gambiae populations with a mortality rate of $100 \%$ when exposed to clothianidin dissolved in acetone+MERO at a concentration of $90 \mu \mathrm{g} / \mathrm{ml}$ (Figure 1A). However, when exposed to clothianidin dissolved in acetone alone $(150 \mu \mathrm{g} / \mathrm{ml})$, the mortality varied from $51.1 \% \pm 15.2$ in Nkolondom to $95.9 \% \pm 2.6$ in Njombe-Penja. On the other hand, when using Ethanol as solvent, the mortality varied from $11.4 \% \pm 4.6$ in Nkolondom to $71.05 \% \pm 6.9$ and $89.3 \% \pm 7.6$ in Njombe-penja and Ndjilli (DRC) respectively (Figure 1A). The mortality with these two solvents increased significantly from $24 \mathrm{~h}$ to 7 days post-exposure confirming a slow-acting effect of this insecticide (Figure S1, S2 \& S3).

\section{Susceptibility profile to Imidacloprid}

The susceptibility to imidacloprid was evaluated on mosquitoes from Nkolondom and Mangoum

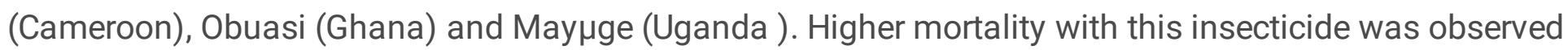
in all the localities when using acetone+MERO as solvent. In Nkolondom, a mortality rate of $83.93 \%$ was observed with acetone + MERO compared to $65.29 \pm 16.63 \%$ with acetone only $\left(X^{2}=10.9 ; P=0.001\right)$ and $27.16 \%$ with ethanol $\left(X^{2}=64.8 ; P<0.0001\right)$ suggesting a likely resistance in this population (Figure $\left.2 A\right)$. The similar pattern was observed in Mangoum where a mortality rate of $96.58 \%$ was observed with acetone + MERO compared to $27.71 \%$ with acetone only $\left(X^{2}=100 ; P<0.0001\right)$ and $40.37 \%$ with absolute ethanol $\left(X^{2}=72.8 ; P<0.0001\right)$ (Figure 2A). In Ghana, imadacloprid dissolved in Acetone + MERO induced $97.92 \%$ mortality and $57.16 \%$ when dissolved in acetone only $\left(X^{2}=47.3 ; P<0.0001\right)$. The mortality was very low when using absolute ethanol as solvent with mortality rate of $12.63 \%\left(X^{2}=146 ; P<0.0001\right)$. The similar profile ( $96.1 \%$ mortality vs $27.3 \%)$ was obtained in Uganda $\left(\chi^{2}=99.6 ; P<0.0001\right)$.

\section{Susceptibility profile to acetamiprid}

When dissolved in acetone+MERO, acetamiprid also displayed greater efficacy compared to when dissolved in acetone only (Figure 2B). In Nkolondom, 100\% mortality was obtained when using acetone and MERO compared to $73.5 \pm 6.6 \%$ without MERO $\left(X^{2}=30.4 ; P<0.0001\right)$. In Obuasi, $89 \pm 4.6 \%$ mortality was obtained when using acetone and MERO compared to $30 \%$ without MERO $\left(X^{2}=71.8 ; P<0.0001\right)$ (Figure 2B). In May $\mu g e$, full susceptibility was noticed with MERO compared to $60 \%$ mortality without MERO $\left(X^{2}=49.7 ; P<0.0001\right)$. These results suggest that the level of reduced susceptibility to acetamiprid is higher in Ghana compared to other locations tested.

\section{Synergist test}

Susceptibility testing using PBO, DEM, and DEF as synergists revealed a significant recovery of susceptibility to clothianidin in Nkolondom which showed a highest level of reduced susceptibility to this 
insecticide when diluted in ethanol or acetone alone (Figure 1B). In Nkolondom, the mortality with clothianidin + PBO was $96.01 \pm 2.6 \%$ versus $51.9 \pm 8.5 \%$ for clothianidin without PBO pre-exposure $\left(\chi^{2}=\right.$ $59.5 ; p<0.0001)$. The same pattern was observed with DEM: DEM pre-exposure $92.1 \pm 3.2 \%$ vs. $51.9 \pm$ $8.5 \%$ for no DEM pre-exposure $\left(\chi^{2}=38.6 ; p<0.0001\right)$ (Figure 1B). Synergist bioassay with DEF also revealed significant recovery of susceptibility to clothianidin (DEF pre-exposure $86.4 \pm 7.1 \%$ versus $51.9 \pm$ $8.5 \%$ mortality without DEF pre-exposure $\left(X^{2}=28.6 ; p<0.0001\right)$ although this was slightly lower compared to PBO and DEM. All these results suggest that monooxygenases, GSTs and esterases all combined to drive the reduced susceptibility clothianidin resistance in Nkolondom.

\section{Diagnostic dosage of clothianidin using acetone and MERO as a solvent}

Because of the very high mortality consistently observed with clothianidin dissolved in acetone + MERO, we decided to establish the diagnostic concentration using the susceptible lab strain Kisumu. The mortality $24 \mathrm{~h}$ post exposure to clothianidin were ranged from $66.8 \% \pm 7.0$ at $0.25 \mu \mathrm{g} / \mathrm{ml}, 60.9 \% \pm 9.4$ at $0.5 \mu \mathrm{g} / \mathrm{ml}, 82.8 \% \pm 9.3$ at $1 \mu \mathrm{g} / \mathrm{ml}$ to $94.2 \% \pm 3.6$ at $2 \mu \mathrm{g} / \mathrm{ml}$ and $100 \%$ at $4 \mu \mathrm{g} / \mathrm{ml}, 40 \mu \mathrm{g} / \mathrm{ml}$ and $90 \mu \mathrm{g} / \mathrm{ml}$ (Figure 3). These results suggest that the concentration of $8-10 \mu \mathrm{g} / \mathrm{ml}(2 x$ the dose that kills $100 \%$ the susceptible strain) could be used as a diagnostic dose for monitoring of resistance in the field population of malaria vectors.

\section{Cross-resistance between clothianidin and pyrethroids}

The distribution of L1014F-Kdr_W genotypes in mosquitoes alive after exposure to a sub-lethal dose of clothianidin was as follows: $24.1 \%$ (7/29) homozygous resistant (1014F/F), 27.6\% (8/29) heterozygotes (L1014F-RS) and 55.17\% (16/29) homozygous susceptible (L/L1014) (Figure 4). In the dead mosquitoes, $30.0 \%$ (9/30) were homozygous resistant (1014F/F), 46.7\% (14/30) were heterozygotes (L1014F-RS) whereas 23.3\% (7/30) were homozygous susceptible (L/L1014). A significant difference was observed in the distribution of L1014F-Kdrw genotypes between alive and dead mosquitoes $\left(X^{2}=18.5 ; P<0.0001\right)$ with the homozygote resistant mosquitoes less able to survive (OR=0.5; IC 95\%: 0.3-0.9; $P=0.02$ ) compared to the susceptible genotypes (Table 1). In contrast, a significant difference was observed in the distribution of the I114T-GSTe2 genotypes between dead and alive mosquitoes and those with resistant allele had more ability to survive clothianidin exposure $\left(\chi^{2}=9.78 ; P=0.007\right)$ (Figure 4). Assessing of the odds-ratio confirmed that mosquitoes with the $114 \mathrm{~T}$ resistant allele have a significantly greater ability to survive compared to those with the 1114 allele as homozygous resistant mosquitoes were significantly more likely to survive clothianidin exposure compared to both heterozygote (OR=2.10; IC 95\%: 1.11-3.97; $\mathrm{P}=0.013$ ) and homozygous susceptible (OR=2.46; IC 95\%: 1.15-5.26; $\mathrm{P}=0.012)$ mosquitoes (Table 2). There was no difference between heterozygote and susceptible mosquitoes ( $O R=1.17 ; P=0.41)$.

\section{Discussion}

The present study compared the efficacy of neonicotinoids in the major malaria vector An. gambiaes.I across many African countries using three different solvents. Susceptibility testing confirmed that 
neonicotinoids are slower-acting insecticides (when using acetone or ethanol alone) compared with neurotoxic pyrethroids [17]. While pyrethroids are characterized by rapid mortality of mosquitoes within 24 hours, clothianidin/imidacloprid/acetamiprid dissolved in ethanol or acetone alone induced mortality at $24 \mathrm{~h}$ post-exposure was generally low (albeit highly variable) and increased over days. Final mortality was recorded seven days post-exposure when using ethanol/acetone alone as done previously [10]. The mortality was low when using ethanol and acetone alone and was the lowest in Nkolondom, an area of intense agriculture. The mortality rates of less than $20 \%$ with ethanol and less than $40 \%$ with acetone observed in this locality support a recent report of reduced susceptibility to neonicotinoids in the $A n$. gambiae population of this location [18]. However, the addition of 1000ppm MERO® (81\% Rapeseed oil methyl ester) increased significantly the effect of these insecticides with $100 \%$ mortality for all the populations $24 \mathrm{~h}$ post exposure even at a very low concentration. The low knock-down/mortality observed for all the populations when using ethanol or acetone alone as a solvent could be linked to the crystallization issue reported for neonicotinoids [19] preventing complete knockdown/mortality within 1$2 \mathrm{~h}$. The use of MERO ${ }^{\circledR}$ by preventing the crystallization, keeps the neonicotinoids for a longer period in a soluble state and increases the reliability of the bottle assay.

Despite the low mortality obtained with acetone or ethanol alone, a significant difference was observed between different populations with a mortality rate of $11-36 \%$ observed in Nkolondom, $31.07 \%$ in Mangoum $45.59 \%$ in Ghana and Uganda compared to $71 \%$ in Njombe or $89.3 \%$ in Congo and $100 \%$ in Kisumu. This indicates that using ethanol or acetone alone might still be useful in capturing the variability between populations and even to detect those populations with reduced susceptibility and thus allow a better management of resistance. In contrast, using acetone plus MERO although more suitable in showing the full efficacy of neonicotinoids, might mask the selection of resistance in populations if not used at the right dose and could prevent detecting the emergence of resistance. Therefore, to take advantage of the strengths of both profiles it could be beneficial to monitor the susceptibility profile to neonicotinoids using acetone (or ethanol) alone and also acetone plus MERO. Because the dose of $90 \mu \mathrm{g} / \mathrm{ml}$ as recommended by Bayer or $50 \mu \mathrm{g}$ as determined recently in Ivory coast is very high for monitoring of resistance, one option could be to reduce this dose to a level allowing to assess the efficacy of neonicotinoids while monitoring resistance development. In this study we observed that the concentration of $8-10 \mu \mathrm{g} / \mathrm{ml}$ could be used for monitoring of resistance to clothianidin as $4 \mu \mathrm{g}$ was the minimum concentration giving $100 \%$ mortality with the susceptible lab strain Kisumu.

\section{Cross resistance between Pyrethroid and neonicotinoid}

A negative association was observed between the 1014F-kdr allele and resistance to clothianidin. Such negative impact could be attributed to the deleterious effect of kdr or other related genes in the presence of clothianidin, for which the vgsc is not the target. Accordingly, a gradual decrease of kdr-resistant homozygotes mosquitoes was observed during the clothianidin selection process by Zoh et (2021) and this could explain the negative correlation observed in this study between $\mathrm{kdr}$ and clothianidin resistance. This is the first time such negative association is observed between pyrethroid resistance mechanism and insecticides with different mode of action. 
Synergist assay using PBO and DEF also revealed significant recovery of susceptibility to clothianidin showing that monoxygenases, GSTs and esterases are all involved in clothianidin resistance. Over expression of P450s monoxygenases was frequently reported in many resistant cases such as in $A n$ gambiae recently where a strong selection signature associated with clothianidin selection was observed on a cytochrome P450 gene cluster with the gene CYP6M1 showing the highest selection signature together with a transcription profile supporting a role in clothianidin resistance. Overexpression of P450s were also mentioned in M. persicae [20], Bemisia tabaci [21-23], Trialeurodes vaporariorum [24], Nilaparvata I $\mu$ gens [25], Leptinotarsa decemlineata [26] and many other pests [27].

For the first time, we observed in this study a significant correlation between GSTe2 and clothianidin resistance in An. gambiae indicating that GSTe2 could metabolise or could be involved in phase 2 conjugation of metabolite of clothianidin degradation. This can be confirmed by the synergist testing with DEM which helped to recover the susceptibility to clothianidin. Since GSTe2 is also pyrethroid/DDT resistance gene, its association with clothianidin resistance indicates that clothianidin-based tools could rapidly lose their efficacy in areas of high GSTe2-based metabolic resistance. However, future studies are needed to establish the role of GST or specific mechanisms such as esterases and P450s in clothianidin resistance.

\section{Conclusion}

This study investigated the susceptibility of the major African malaria vector An. gambiae to neonicotinoids using three different solvents and evaluated potential cross resistance between pyrethroid resistance markers and clothianidin survival. The study revealed that the use of acetone or ethanol alone as a solvent for clothianidin can over-estimate the level of resistance in mosquitoes due to crystallisation issue. Furthermore, we showed that the use of clothiandin dissoved in acetone + MERO display very strong efficacy with $2-4 \mu \mathrm{g} / \mathrm{ml}$ as diagnostic dose for monitoring of resistance to clothianidin but could mask the development of resistance. We recommend monitoring the susceptibility using both acetone alone and Acetone+MERO to capture the accurate resistance profile of the mosquito populations.

\section{Declarations}

\section{Availability of data and materials}

All the relevant datasets supporting the conclusions of this article are included within the article.

Author Contributions: C.S.W. conceived and designed the study; M.T, LMJM, B.D.M, D.N.N, collected the samples with the help of J.K, F.W, E.Z.M., and M.O.; M.T., T.A, and W.T, maintained the strain in the insectary and performed bioassays; M.T. and T.A performed the molecular analyses; M.T, and C.S.W. analyzed the data; M.T wrote the manuscript with contributions from and C.S.W., J.K, F.W, E.Z.M., and M.O.. All authors read and approved the final manuscript.

\section{Acknowledgements}

Page 10/18 
We are very grateful to all the international partners for supporting in sample collection. We are also grateful to the Community Health Workers from all the villages

Funding: This study was funded by the BMGF Grant (INV-006003) awarded to CSW.

Author information

Centre for Research in Infectious Diseases (CRID), P.O. Box 13501, Yaoundé, Cameroon

Magellan TCHOUAKUI, Tatiane ASSATSE, Leon M. J. MUGENZI, Benjamin D. MENZE, Daniel NguiffoNGUETE, Williams TCHAPGA \& Charles S. WONDJI

Entomology department, Uganda Virus Research Institute (UVRI), P.O.Box 49 Entebbe, Uganda Jonathan KAYONDO,

Institut National de Recherche Biomédicale, Kinshasa, Democratic Republic of Congo

Francis WATSENGA \& Emile Zola MANZAMBI

${ }^{4}$ Radiation Entomology and Pest Management Centre, Biotechnology and Nuclear Agriculture Research Institute, Ghana Atomic Energy Commission, Ghana, P.O. Box LG80, Legon-Accra, Ghana.

Michael OSAE

Department of Vector Biology, Liverpool School of Tropical Medicine, Pembroke Place, L35QA, Liverpool, UK

Charles S. WONDJI

International Institute of Tropical Agriculture (IITA), Yaoundé, P.O. Box 2008, Cameroon

Charles S. WONDJI

Parasitology and Ecology Laboratory, Department of Animal Biology and Physiology, Faculty of Science, P.O. Box 812, University of Yaoundé 1, Yaoundé, Cameroon

Tatiane ASSATSE

Ethics declarations: NA

Consent to publication: This paper is published with the authority from the Executive Director of the Centre for Research in Infectious Diseases (CRID)

Conflicts of Interest: The authors declare no conflicts of interests. 


\section{References}

1. Bhatt S, Weiss DJ, CaMEROn E, Bisanzio D, Mappin B, Dalrymple U, et al. The effect of malaria control on Plasmodium falciparum in Africa between 2000 and 2015. Nature. 2015;526(7572):20711.

2. WHO. World Malaria Report 2016. Geneva: World Health Organization; 2016.

3. Riveron JM, Tchouakui M, Mugenzi L, Menze BD, Chiang M-C, Wondji CS. Insecticide resistance in malaria vectors: An update at a global scale. Towards Malaria Elimination-A Leap Forward: IntechOpen; 2018.

4. Hemingway J, Vontas J, Poupardin R, Raman J, Lines J, Schwabe C, et al. Country-level operational implementation of the Global Plan for Insecticide Resistance Management. Proc Natl Acad Sci U S A. 2013;110(23):9397-402.

5. Blaschke TF, Lumpkin M, Hartman D. The World Health Organization prequalification program and clinical pharmacology in 2030. Clin Pharmacol Ther. 2020;107(1):68-71.

6. Hemingway J. The way forward for vector control. Science. 2017;358(6366):998-9.

7. WHO. Global vector control response: progress in planning and implementation. 2020.

8. Krupke $\mathrm{CH}$, Long EY. Intersections between neonicotinoid seed treatments and honey bees. Current opinion in insect science. 2015;10:8-13.

9. Yamamoto I, Casida JE. Nicotinoid insecticides and the nicotinic acetylcholine receptor. 1999.

10. Oxborough RM, Seyoum A, Yihdego Y, Chabi J, Wat'senga F, Agossa FR, et al. Determination of the discriminating concentration of chlorfenapyr (pyrrole) and Anopheles gambiae sensu lato susceptibility testing in preparation for distribution of Interceptor ${ }^{\circledR} \mathrm{G} 2$ insecticide-treated nets. Malar J. 2021;20(1):1-10.

11. Agumba S, Gimnig JE, Ogonda L, Ombok M, Kosgei J, Munga S, et al. Diagnostic dose determination and efficacy of chlorfenapyr and clothianidin insecticides against Anopheles malaria vector populations of western Kenya. Malar J. 2019;18(1):1-9.

12. Dagg K, Irish S, Wiegand RE, Shililu J, Yewhalaw D, Messenger LA. Evaluation of toxicity of clothianidin (neonicotinoid) and chlorfenapyr (pyrrole) insecticides and cross-resistance to other public health insecticides in Anopheles arabiensis from Ethiopia. Malar J. 2019;18(1):1-11.

13. Darriet F, Chandre F. Efficacy of six neonicotinoid insecticides alone and in combination with deltamethrin and piperonyl butoxide against pyrethroid-resistant Aedes aegypti and Anopheles gambiae (Diptera: Culicidae). Pest management science. 2013;69(8):905-10.

14. Mouhamadou CS, de Souza SS, Fodjo BK, Zoh MG, Bli NK, Koudou BG. Evidence of insecticide resistance selection in wild Anopheles coluzzii mosquitoes due to agricultural pesticide use. Infectious diseases of poverty. 2019;8(1):1-8.

15. Bass C, Williamson MS, Wilding CS, Donnelly MJ, Field LM. Identification of the main malaria vectors in the Anopheles gambiae species complex using a TaqMan real-time PCR assay. Malar J. 2007;6(1):1-9. 
16. Mitchell SN, Rigden DJ, Dowd AJ, Lu F, Wilding CS, Weetman D, et al. Metabolic and target-site mechanisms combine to confer strong DDT resistance in Anopheles gambiae. PloS one. 2014;9(3):e92662.

17. Anto F, Asoala V, Anyorigiya T, Oduro A, Adjuik M, Owusu-Agyei S, et al. Insecticide resistance profiles for malaria vectors in the Kassena-Nankana district of Ghana. Malaria Journal. 2009;8(1):1-8.

18. Fouet C, Ashu AF, Ambadiang MM, Tchapga W, Wondji CS, Kamdem C. Resistance of Anopheles gambiae to the new insecticide clothianidin associated with unrestricted use of agricultural neonicotinoids in Yaounde, Cameroon. BioRxiv. 2020.

19. Stica C, Jeffries CL, Irish SR, Barry Y, Camara D, Yansane I, et al. Characterizing the molecular and metabolic mechanisms of insecticide resistance in Anopheles gambiae in Faranah, Guinea. Malar J. 2019;18(1):1-15.

20. Puinean AM, Foster SP, Oliphant L, Denholm I, Field LM, Millar NS, et al. Amplification of a cytochrome $\mathrm{P} 450$ gene is associated with resistance to neonicotinoid insecticides in the aphid Myzus persicae. PLOS genetics. 2010;6(6):e1000999.

21. Karunker I, Benting J, Lueke B, Ponge T, Nauen R, Roditakis E, et al. Over-expression of cytochrome P450 CYP6CM1 is associated with high resistance to imidacloprid in the $B$ and $Q$ biotypes of Bemisia tabaci (Hemiptera: Aleyrodidae). Insect Biochem Mol Biol. 2008;38(6):634-44.

22. Jones CM, Daniels M, Andrews M, Slater R, Lind RJ, Gorman K, et al. Age-specific expression of a P450 monooxygenase (CYP6CM1) correlates with neonicotinoid resistance in Bemisia tabaci. Pestic Biochem Physiol. 2011;101(1):53-8.

23. Yang N, Xie W, Jones C, Bass C, Jiao X, Yang X, et al. Transcriptome profiling of the whitefly B emisia tabaci reveals stage-specific gene expression signatures for thiamethoxam resistance. Insect Mol Biol. 2013;22(5):485-96.

24. Karatolos N, Williamson MS, Denholm I, Gorman K, Ffrench-Constant RH, Bass C. Over-expression of a cytochrome $\mathrm{P} 450$ is associated with resistance to pyriproxyfen in the greenhouse whitefly Trialeurodes vaporariorum. PLoS One. 2012;7(2):e31077.

25. Bass C, Carvalho R, Oliphant L, Puinean A, Field L, Nauen R, et al. Overexpression of a cytochrome P450 monooxygenase, CYP6ER1, is associated with resistance to imidacloprid in the brown planthopper, Nilaparvata I $\mu$ gens. Insect molecular biology. 2011;20(6):763-73.

26. Zhao J-Z, Bishop BA, Grafius EJ. Inheritance and synergism of resistance to imidacloprid in the Colorado potato beetle (Coleoptera: Chrysomelidae). Journal of economic entomology. 2000;93(5):1508-14.

27. Bass C, Denholm I, Williamson MS, Nauen R. The global status of insect resistance to neonicotinoid insecticides. Pestic Biochem Physiol. 2015;121:78-87.

\section{Tables}


Table 1. Assessment of the association between L1014F-Kdr genotypes/alleles and the ability of mosquitoes to survive clothianidin exposure. SS: homozygote susceptible; RR: homozygote resistant; RS: heterozygote; $(*)$ significant difference.

\begin{tabular}{|lll|}
\hline Genotypes & \multicolumn{2}{c|}{ L1014F-Kdr and clothianidin resistance } \\
\cline { 2 - 3 } & Odds ratio & $p$-value \\
RR vs. SS & $\begin{array}{l}0.3 \\
(0.2-0.7)\end{array}$ & $0,002^{\star \star}$ \\
RR vs. RS & $\begin{array}{l}1.4 \\
(0.7-2.8)\end{array}$ & 0.23 \\
\hline RS vs. SS & $\begin{array}{l}0.2 \\
(0.1-0.5)\end{array}$ & $<0.0001^{\star \star *}$ \\
\hline R vs. S & $\begin{array}{l}0.5 \\
(0.3-0.9)\end{array}$ & $0.02^{\star}$ \\
\hline
\end{tabular}

Table 2. Assessment of the association between I114T-GSTe2 genotypes/alleles and the ability of mosquitoes to survive clothianidin exposure. SS: homozygote susceptible; RR: homozygote resistant; RS: heterozygote; $\left(^{\star}\right)$ significant difference.

\begin{tabular}{|c|c|c|}
\hline \multirow[t]{3}{*}{ Genotypes } & \multirow{2}{*}{\multicolumn{2}{|c|}{ I114T-GSTe2 and clothianidin resistance }} \\
\hline & & \\
\hline & Odds ratio & $p$-value \\
\hline RR vs. SS & $\begin{array}{l}4.51 \\
(1.3-15.4)\end{array}$ & $0,0006^{\star \star}$ \\
\hline RR vs. RS & $\begin{array}{l}2.5 \\
(1.2-4.9)\end{array}$ & $0.01 *$ \\
\hline RS vs. SS & $\begin{array}{l}1.8 \\
(0.6-5.6)\end{array}$ & 0.4 \\
\hline R vs. S & $\begin{array}{l}1.6 \\
(0.9-2.9)\end{array}$ & $0.05^{\star}$ \\
\hline
\end{tabular}

\section{Figures}



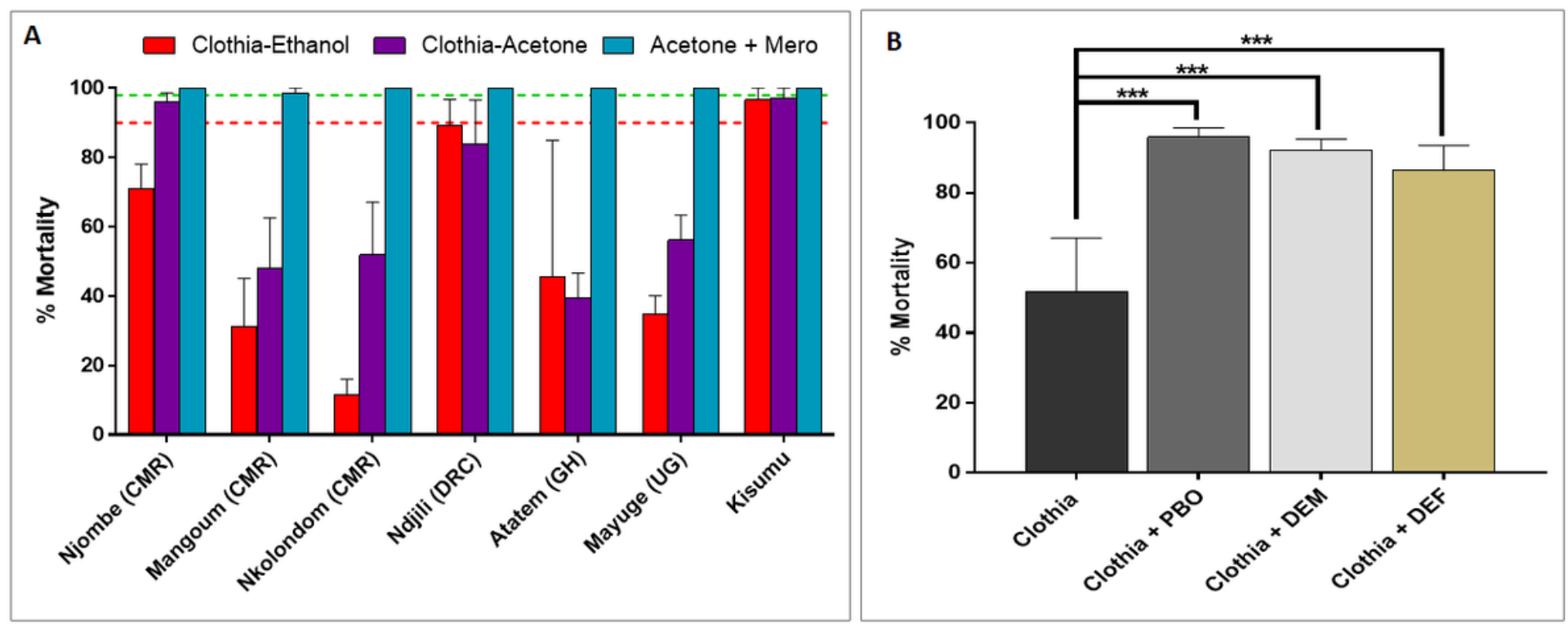

Figure 1

\section{Susceptibility profile of An. gambiae s./ to clothianidin across Africa}

(A) Mortality rate of mosquitoes from various sites 7 days post-exposure to clothianidin dissolved in different solvents compared to the susceptible lab strain Kisumu. (B) Effect of pre-exposure to synergist PBO, DEM and DEF against clothianidin on An. gambiae from Nkolondom. Results are average of percentage mortalities from four-five replicates each. The bars represent the standard error on the mean (SEM), linear color dots indicate the threshold for resistance (red) and susceptibility (green). CMR: Cameroon; DRC: Democratic Republic of Congo; GH: Ghana; UG: Uganda .
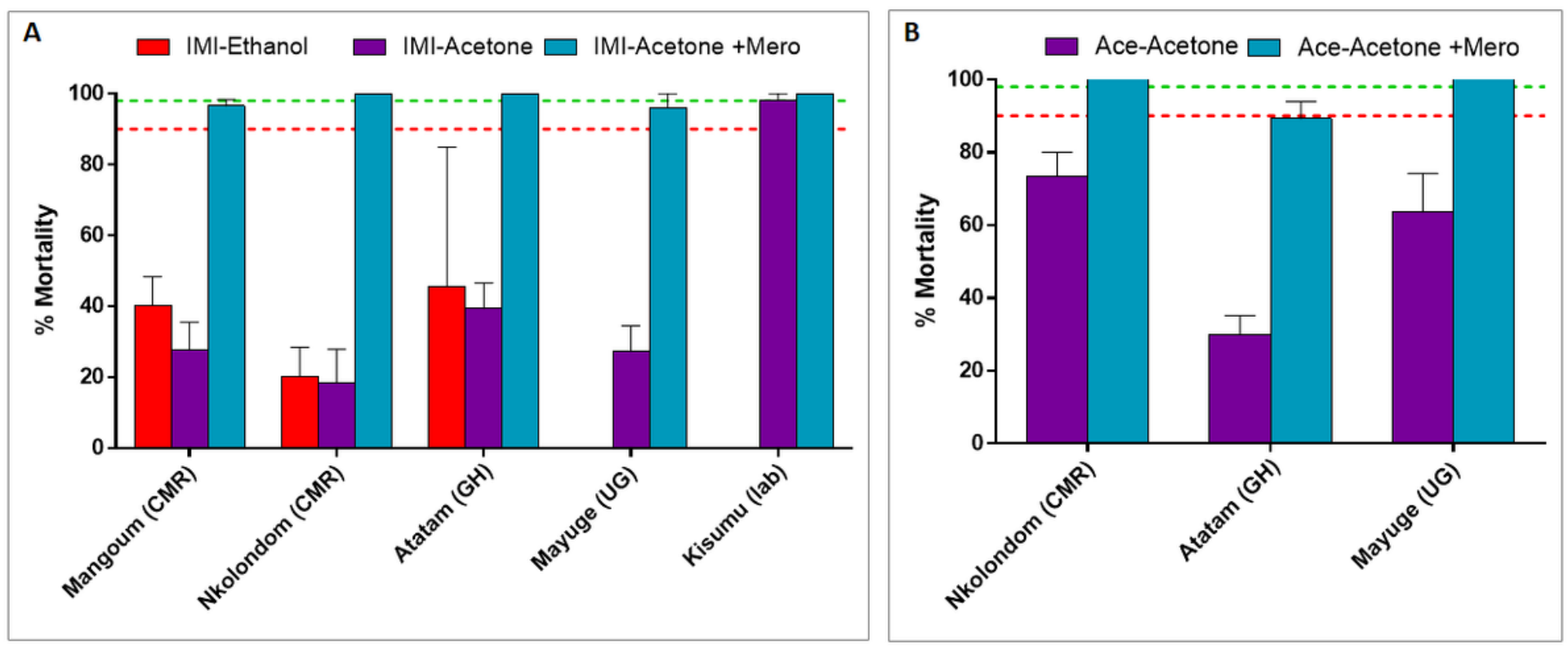

Figure 2 
Susceptibility profile of $\boldsymbol{A n}$. gambiae s.I to imidacloprid and acetamiprid across Africa. Mortality rate of mosquitoes from different sites 7 days post-exposure to imidacloprid (A) and acetamiprid (B) dissolved in various solvents compared to the susceptible lab strain Kisumu. Results are average of percentage mortalities from four-five replicates each \pm SEM. Linear colour dots indicate the threshold for resistance (red) and susceptibility (green). CMR: Cameroon; GH: Ghana; UG: Uganda .

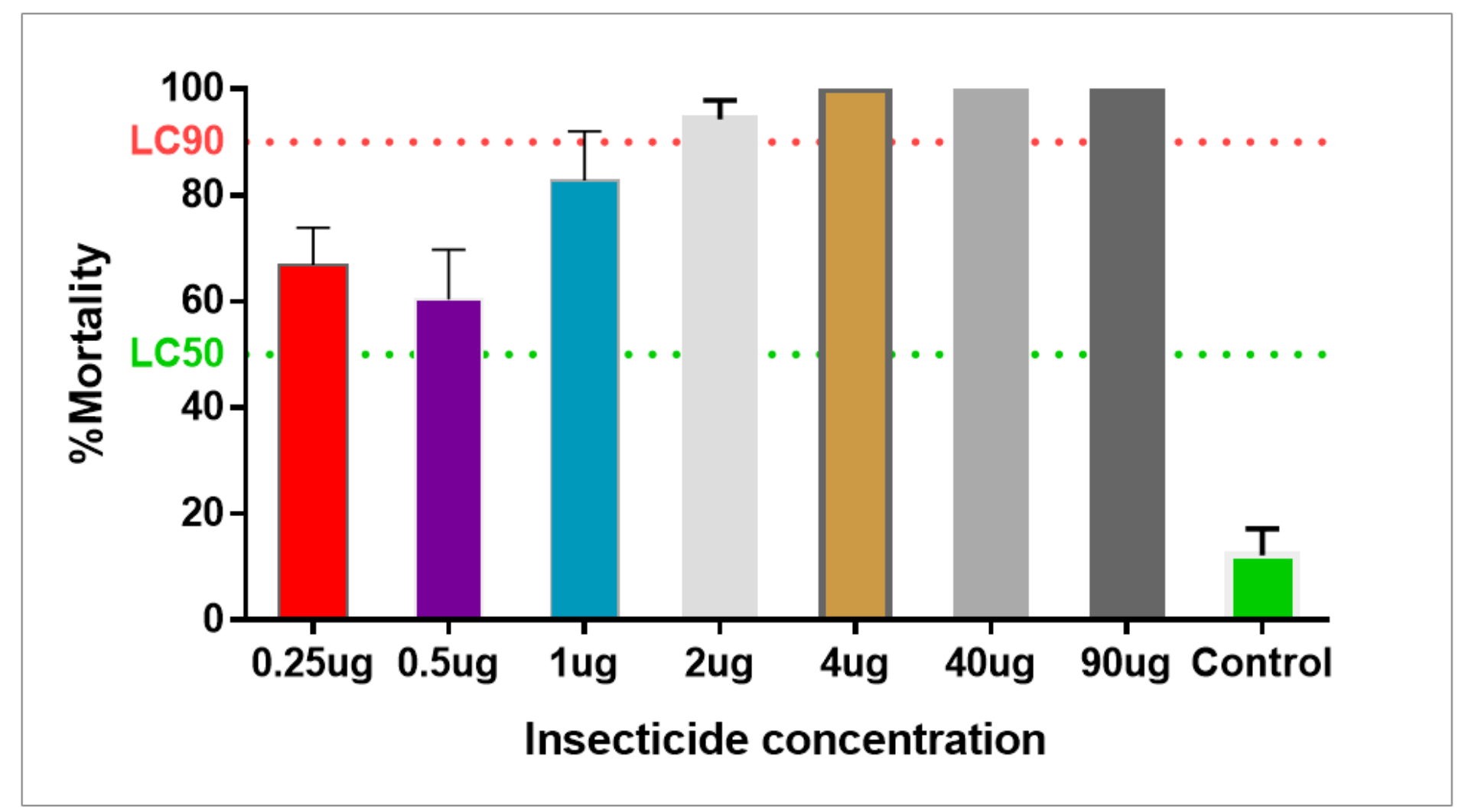

Figure 3

Assessment of diagnostic dose of clothianidin using acetone and MERO as solvent. Percentage mortality (24 h) of the susceptible lab strain Kisumu after exposure to each of the six concentrations of clothianidin (with acetone+MERO as solvent). LC50 represents the concentration able to kill $50 \%$ of mosquitoes and LC90 the concentration able to kill $90 \%$. 

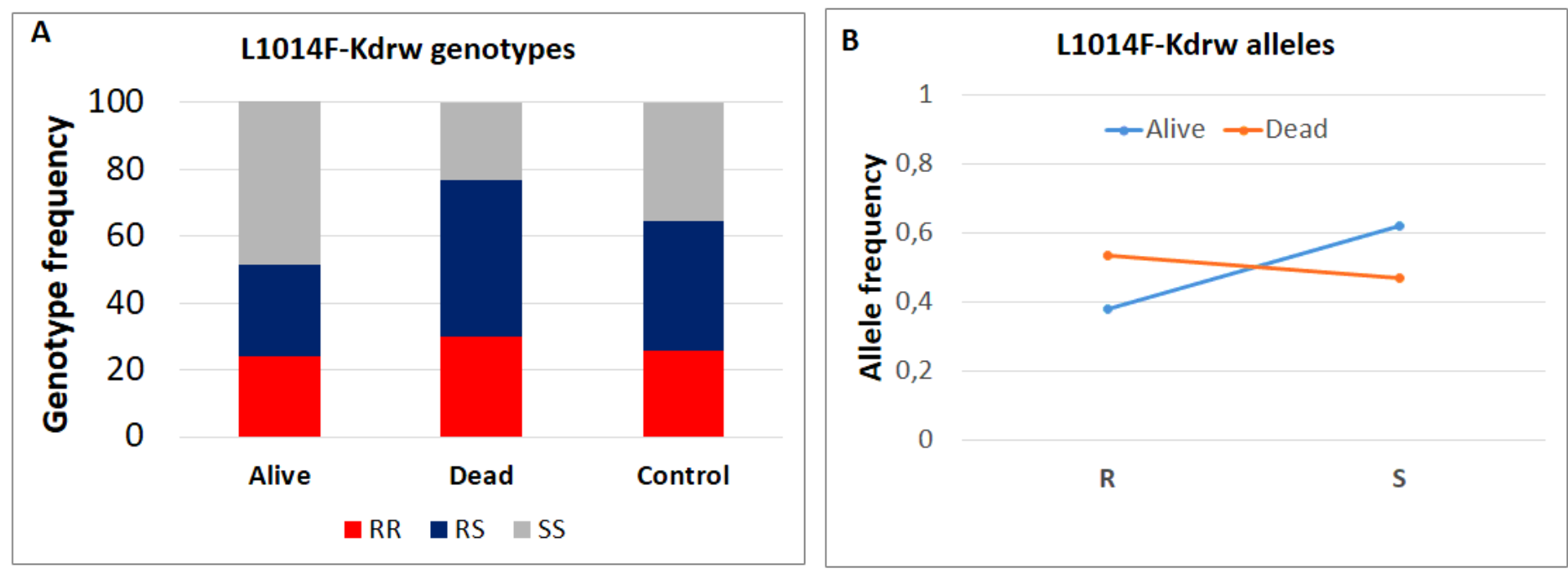

Figure 4

Association between the L1014F-kdr_w mutation and resistance to clothianidin. Distribution of genotypes (A) and alleles (B) among the dead and alive mosquitoes after exposure to clothianidin. $\mathrm{R}$ represents the 1014F-resistant allele while S represent the L1014 susceptible allele.

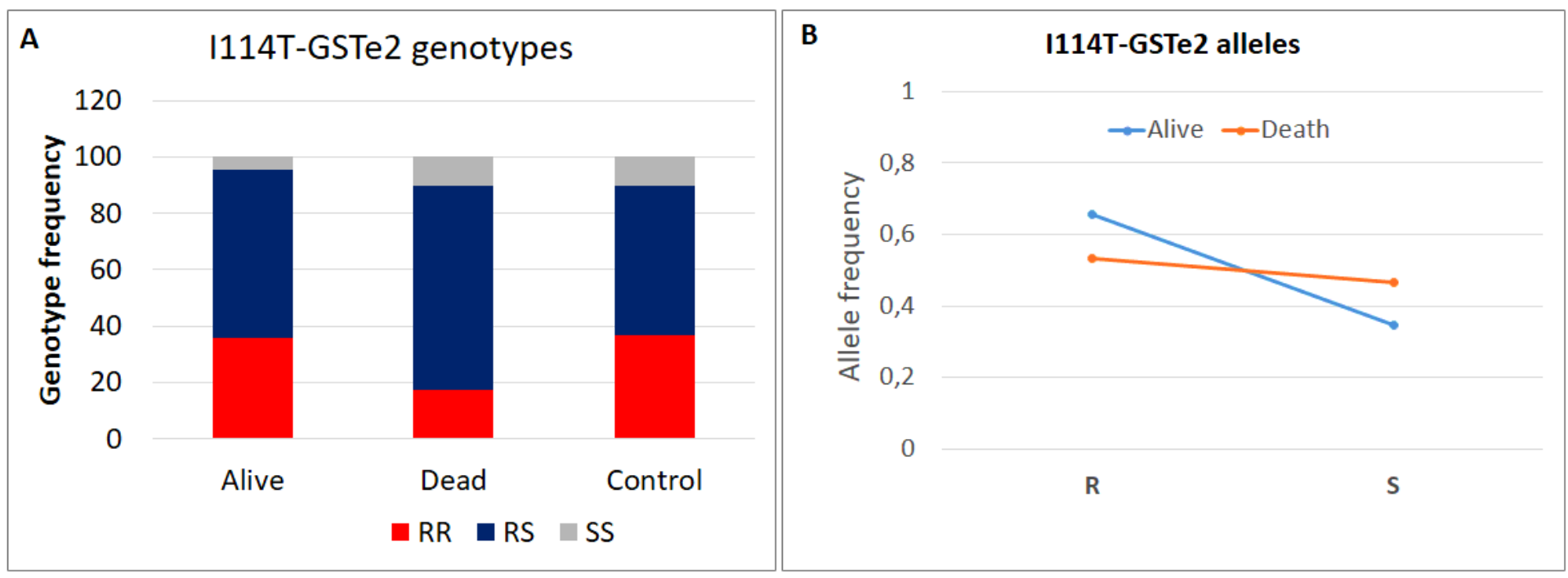

Figure 5

Association between the 1114TF-GSTe2 mutation and resistance to clothianidin. Distribution of genotypes (A) and alleles (B) among the dead and alive mosquitoes after exposure to clothianidin. $R$ represents the 114T-resistant allele while $S$ represents the I114 susceptible allele.

\section{Supplementary Files}

This is a list of supplementary files associated with this preprint. Click to download. 
- FigureS1.tif

- Figures2.tif

- Figures3.tif 